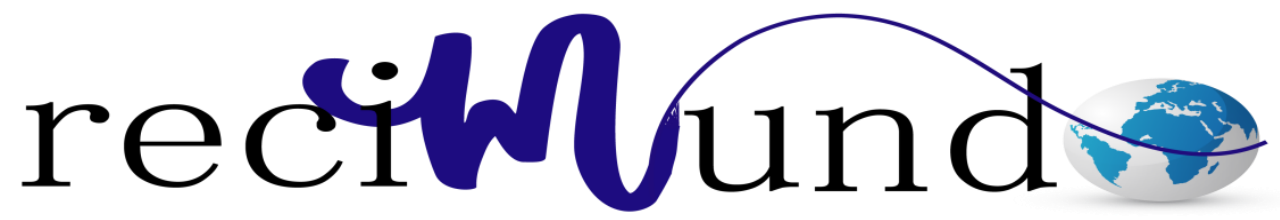

Revista Científica Mundo de la Investigación y el Conocimiento

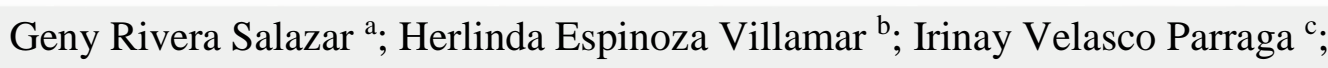
Roció Suarez Fuentes ${ }^{d}$

Factores socioculturales asociados a la interrupción de la lactancia materna exclusiva en madres con niños menores de 6 meses

Revista Científica Mundo de la Investigación y el Conocimiento. Vol. 2 núm., 1, febrero, ISSN: 2588-073X, 2018, pp. 3-25

DOI: $10.26820 /$ recimundo/2.1.2018.3-25

Editorial Saberes del Conocimiento

Recibido: 05/12/2017

Aceptado: 10/02/2018

a. Universidad Católica Santiago de Guayaquil; geny.rivera@cu.ucsg.edu.ec

b. Junta de Beneficencia de Guayaquil; Complejo Hospitalario Alejandro Mann; Hospital de Niños "Dr. Roberto Gilbert Elizalde"; hespinoza@jbgye.org.ec

c. Junta de Beneficencia de Guayaquil; Complejo Hospitalario Alejandro Mann; Hospital de Niños "Dr. Roberto Gilbert Elizalde"; ivelasco@jbgye.org.ec

d. Junta de Beneficencia de Guayaquil; Complejo Hospitalario Alejandro Mann; Hospital de Niños "Dr. Roberto Gilbert Elizalde"; rsuarez@jbgye.org.ec 


\section{Factores socioculturales asociados a la interrupción de la lactancia materna exclusiva en madres con niños menores de 6 meses}

Vol. 2, núm. 1., (2018)

Geny Rivera Salazar; Herlinda Espinoza Villamar; Irinay Velasco Parraga; Roció Suarez Fuentes

\section{RESUMEN}

Esta investigación tiene como objetivo determinar factores socioculturales que se asocian a la interrupción de la lactancia materna exclusiva en madres con niños menores de seis meses de edad en un hospital pediátrico. Fundamentada con la teoría de Madeleine Leininger, enfermería transcultural que se asocia con las ciencias sociales y antropología dando gran relevancia a la interculturalidad, concibiendo a la enfermería como una ciencia netamente humanística encaminada al cuidado holístico del ser humano y que al planificar los cuidados se considere su cultura entendiendo que se trata de un ser complejo y que estos cuidados sean aceptados. El enfoque es mixto, Cuali-Cuantitativo. Cualitativo seleccionando un grupo focal quienes firmaron un consentimiento informado y Cuantitativo aplicando una encuesta, es un trabajo de campo, descriptivo, explicativo y de corte transversal, cuya modalidad de estudio es análisis-síntesis, inductivo-deductivo. Analizando el núcleo del sentido, los resultados cuantitativos y la fundamentación de la teórica Madeleine, Se concluyó que los factores económicos, diferentes situaciones familiares, estilos de vidas y costumbres, repercutieron en el abandono o continuidad de la lactancia materna exclusiva.

Palabras clave: Enfermería, madres, lactancia materna exclusiva. 


\title{
Factores socioculturales asociados a la interrupción de la lactancia materna exclusiva en madres con niños menores de 6 meses
}

Vol. 2, núm. 1., (2018)

Geny Rivera Salazar; Herlinda Espinoza Villamar; Irinay Velasco Parraga; Roció Suarez Fuentes

\begin{abstract}
This research aims to determine sociocultural factors that are associated with the interruption of exclusive breastfeeding in mothers with children under six months of age in a pediatric hospital. Based on the theory of Madeleine Leininger, cross-cultural nursing that is associated with the social sciences and anthropology giving great relevance to interculturality, designing nursing as a purely humanistic science aimed at the holistic care of the human being and that when planning care is considered their culture understanding that it is a complex being and that these cares are accepted. The approach is mixed, Quali-Quantitative. Qualitative selecting a focus group who signed an informed and quantitative consent applying a survey, is a fieldwork, descriptive, explanatory and cross-sectional, whose mode of study is analysis-synthesis, inductive-deductive. Analyzing the core of meaning, quantitative results and the foundation of the theoretical Madeleine, It was concluded that economic factors, different family situations, lifestyles and customs, impacted on the abandonment or continuity of exclusive breastfeeding.
\end{abstract}

Keywords: Nursing, mothers, exclusive breastfeeding. 


\section{Factores socioculturales asociados a la interrupción de la lactancia materna exclusiva en madres con niños menores de 6 meses}

Vol. 2, núm. 1., (2018)

Geny Rivera Salazar; Herlinda Espinoza Villamar; Irinay Velasco Parraga; Roció Suarez Fuentes

\section{Introducción.}

La Organización Mundial de la Salud (OMS) y el Fondo de las Naciones Unidas para la Infancia (UNICEF), reconocen y recomiendan la práctica de la lactancia materna exclusiva durante los primeros seis meses de vida, puesto que la lactancia materna es la forma ideal de aportar a los niños los nutrientes necesarios para su adecuado crecimiento y desarrollo. (1)

Diversos autores indican que el amplio abandono de la Leche Materna es una característica del siglo XX, según Mead Y Newton (1997), Winnikoff y Cols (1996), parece ser que influyen más los factores culturales que las capacidades biológicas de las mujeres al igual que factores socioeconómicos y familiares. Ysunsa (1992) menciona que la escolaridad y la ubicación de las familias lactantes guardan relación con el mantenimiento de la Lactancia Materna. Van Esterik y Cols (1994) enuncian como los principales factores socioeconómicos para la suspensión de la lactancia materna exclusiva, el ingreso creciente de las mujeres al mercado laboral, las presiones de la vida urbana de las grandes ciudades con su consecuente disminución del tiempo que las madres necesitan dedicarle a sus hijos para mantener la lactancia materna y, la falta de apoyo familiar para solucionar esta problemática. (2)

Haciendo referencia en cuanto a los recursos, la OMS menciona que en los lugares de escasos recursos, donde habitualmente hay falta de condiciones de sanidad y agua potable, la lactancia materna puede salvar la vida de los recién nacidos; se ha demostrado que los lactantes alimentados con leche materna exclusiva durante los primeros seis meses de vida, tienen diferentes patrones de crecimiento con respecto a los lactantes alimentados con leche de fórmula, 


\section{Factores socioculturales asociados a la interrupción de la lactancia materna exclusiva en madres con niños menores de 6 meses}

Vol. 2, núm. 1., (2018)

Geny Rivera Salazar; Herlinda Espinoza Villamar; Irinay Velasco Parraga; Roció Suarez Fuentes

además la lactancia materna tiene beneficios positivos a largo plazo, incluida una posible protección contra la obesidad. (3)

En la actualidad a pesar de las recomendaciones de la OMS la lactancia materna exclusiva la mantienen hasta los 3 meses un $70 \%$ y de este porcentaje es practicado un $48 \%$ hasta los 6 meses de edad de manera exclusiva.

En publicaciones realizadas se menciona que el consumo de LME ha disminuido y el consumo de leche de fórmula va en aumento, esta situación trae consigo repercusiones tanto económicas y sociales, cuyas tendencias se constituyen en un importante problema de salud pública. La OMS publicó que en América Latina durante los años 2006 hasta el año 2012 la LME bajó de un $22.3 \%$ a un $14.5 \%$. $(4,5)$

Existen muchas condiciones de los países como de las regiones, sí como situaciones políticas tendientes al mejoramiento de los programas de inicio de la lactancia materna inmediata dentro de la primera hora del nacimiento. Según un reporte de los Centros de Control y Prevención de Enfermedades. (CDC) USA refiere que el 51.8\% de las madres mantiene la lactancia materna exclusiva durante los primeros 6 meses de edad y el 30.7\% continua con esta práctica hasta el año de vida, esta información no incluye información desglosada por origen étnico, los CDC manejan cifras según las cuales entre 2000 y 2008 la tasa de mujeres hispanas que empezaron a amamantar a sus bebés recién nacidos aumentó de un $77.6 \%$ a un $80.0 \%$, en comparación con un $71.8 \%$ a $75.2 \%$ entre las blancas. (6)

En publicaciones realizadas de datos correspondientes al año 2015 refiere que en América Latina existe una gran apertura por parte de los gobiernos para el mejoramiento de la LME. 


\section{Factores socioculturales asociados a la interrupción de la lactancia materna exclusiva en madres con niños menores de 6 meses}

Vol. 2, núm. 1., (2018)

Geny Rivera Salazar; Herlinda Espinoza Villamar; Irinay Velasco Parraga; Roció Suarez Fuentes

República Dominicana la LME alcanza un 7\%, México el índice de LME durante los primeros 6 meses de edad se duplicó en los últimos años, al pasar $14.4 \%$ en el año 2012 a $22.3 \%$ en el año 2015, Paraguay con un 24.45, Panamá 27.5\%, Brasil 38.6\%, Ecuador 39.6\%, Colombia 42.8\%, Chile 43.5\%, Argentina 55\% y Perú con la tasa más alta de la región con $68.3 \%$. La tasa global en las Américas de lactancia materna exclusiva es el 38\%. (7)

Ya para el año 2016 en publicaciones para América latina y el Caribe vemos que existen grandes diferencias en cuanto al porcentaje de niños alimentados solo con leche materna durante los primeros seis meses de vida de acuerdo a UNICEF. La tasas de lactancia exclusiva son considerablemente mayores en algunos países, como Bolivia (60\%), Perú (67\%) y Chile (82\%), y muy bajas en otros, como República Dominicana (7\%) y Surinam (3\%). Las tasas de lactancia exclusiva de niños de 6 meses o menos en Brasil (39\%) siguen estando casi 30 puntos porcentuales por debajo de las observadas en Perú y Chile, aunque son considerablemente más altas que las de México (14\%). (8)

En el Ecuador según la encuesta nacional de salud y nutrición años 2011-2013 solo el $43.8 \%$ de los niños y niñas reciben lactancia materna exclusiva durante los primeros 6 meses de vida. Y únicamente el $52 \%$ de los niños recibe leche materna durante su primer mes de vida, porcentaje que se ubica en $48 \%$ para aquellos entre 2 y 3 meses de edad y $34 \%$ para el grupo de entre 4 y 5 meses de edad. Ya para el año 2015 cuenta con una tasa de Lactancia Materna Exclusiva de $39.6 \%$ en menores de 6 meses de edad. Unicef-Ecuador pública que el $77 \%$ de las madres indígenas práctica la lactancia exclusiva hasta antes de los 6 meses, mientras que solo el $23 \%$ de madres montubias lo hacen, presentando la prevalencia más baja de lactancia materna 


\section{Factores socioculturales asociados a la interrupción de la lactancia materna exclusiva en madres con niños menores de 6 meses}

Vol. 2, núm. 1., (2018)

Geny Rivera Salazar; Herlinda Espinoza Villamar; Irinay Velasco Parraga; Roció Suarez Fuentes

exclusiva. La proporción de niños que accedieron a la lactancia materna exclusiva es mayor en el área rural (58\%) en comparación con los niños del área urbana (35\%). El 70\% de los niños de 12 a 15 meses de edad permaneció con la lactancia materna continua en el área rural, mientras que en el área urbana solo lo hizo el 52\%. Únicamente, el 18\% de los niños de 2 años de edad recibió lactancia materna en forma continua. (9)

De acuerdo a los datos estadísticos planteados en el presente estudio se puede observar que la mayor incidencia de las madres que brindaron la lactancia materna exclusiva a sus hijos sobresale en la población rural, probablemente se debe a que estas madres no están incluidas en la producción laboral fuera del hogar, mientras que en el área urbana un gran porcentaje de madres que tienen niños en edad de lactancia materna ayudan a la economía del lugar, por lo que obligatoriamente salen de su hogar. Agregamos a toda esta información que en nuestro país existe el Plan Nacional del Buen Vivir (PNBV) 2013-2017 el mismo que sigue trabajando a nivel gubernamental y se proyecta como país que a finales del año 2017 aumentar la prevalencia de Lactancia Materna Exclusiva durante los primeros 6 meses de vida a un 64\%, a través del MSP y los hospitales amigos. (10)

El (IHAN) se trata de un movimiento mundial propuesto en el año 1991 en respuesta a la Declaración de Innocenti; liderado por la OMS y el fondo de las naciones unidad (UNICEF), se trata de una estrategia mundial para la alimentación del lactante y del niño pequeño cuya finalidad es promover la lactancia materna exclusiva para disminuir la morbimortalidad infantil en todos los establecimientos de salud donde se atienden partos, implementando buenas prácticas, que los gobiernos aseguren que en el sector público y otros protejan, promuevan, 


\section{Factores socioculturales asociados a la interrupción de la lactancia materna exclusiva en madres con niños menores de 6 meses}

Vol. 2, núm. 1., (2018)

Geny Rivera Salazar; Herlinda Espinoza Villamar; Irinay Velasco Parraga; Roció Suarez Fuentes

apoyen la lactancia materna exclusiva de forma continua. En el año 2013 en Ecuador nace la

iniciativa del MSP y de algunos hospitales así como centros de salud de incorporarse a esta iniciativa del "Hospital amigo del niño". Cuya meta es certificarse como tal, la normativa tendiente al mejoramiento de la calidad de la atención per-natal (antes del nacimiento) y de las emergencias obstétricas y neonatales (recién nacidos). También promoverá el parto humanizado, además de la adecuada atención del recién nacido, esto incluye el fomento, apoyo y protección de la lactancia materna exclusiva, y la prevención en la transmisión vertical (madre-hijo/a) del VIH y la sífilis.

El Hospital de niños Dr. Roberto Gilbert Elizalde una institución benéfica, privada que hasta la actualidad no se constituye como un requisito llevar todos los programas del Ministerio de Salud Pública del Ecuador, no cuenta con un área exclusiva destinada para brindar el beneficio de lactancia materna, existe insuficiente información en las salas de hospitalización de los menores de seis meses de edad sobre este tema por parte del personal profesional de enfermería durante toda su estancia hospitalaria. Existe un déficit del profesional de enfermería en el área de consulta externa para brindar información relevante la misma que tenga una fundamentación científica y que llegue a las madres que acuden a este servicio.

A partir del primer semestre del año en curso, como una exigencia del ministerio de salud pública, se constituye un Comité de lactancia materna conformado por profesionales de enfermería del las áreas de neonatología, así como supervisoras de enfermería del Complejo Hospitalario Alejandro Mann, con la finalidad de trabajar inicialmente en las áreas de consulta externa del Hospital Dr. Roberto Gilbert Elizalde y del Hospital de la mujer “Alfredo Paulson” 


\section{Factores socioculturales asociados a la interrupción de la lactancia materna exclusiva en madres con niños menores de 6 meses}

Vol. 2, núm. 1., (2018)

Geny Rivera Salazar; Herlinda Espinoza Villamar; Irinay Velasco Parraga; Roció Suarez Fuentes de la Honorable Junta de Beneficencia de Guayaquil, cuyo centro de atención es brindar a la comunidad en general información oportuna sobre la importancia de la lactancia materna exclusiva durante los primeros seis meses de edad. En el complejo Hospitalario Alejandro Mann en referencia Hospital de niños Dr. Roberto Gilbert Elizalde y Hospital de la mujer Alfredo Paulson, hace aproximadamente seis años docencia en enfermería lleva el programas de educación continua dirigida a la comunidad con diferentes temas de interés de los usuarios, participan los internos de enfermería que según cronograma anual de rotación realizan esta contribución hacia la comunidad, pero en sí, no hace referencia específicamente a temas de lactancia materna exclusiva.

La capacidad de cama que hay en el Hospital Dr. Roberto Gilbert Elizalde para lactantes menores y mayores es de 265 cupos, mientras que en el área de consulta externa se atienden a diario a 600 niños. El Hospital de la mujer Alfredo Paulson fundado en agosto del año 2016, su capacidad de cama es para 400 pacientes, en la actualidad tenemos una disponibilidad vigente de aproximadamente 170 camas ocupadas para toda el área de hospitalización y su atención ambulatoria aproximadamente es de 300 personas por día, de este grupo el $56 \%$ de las usuarias son adolescentes según datos obtenidos del departamento de estadística del complejo hospitalario Alejandro Mann y la jefatura de enfermería.

Y es así, que a través de las observaciones realizadas en el Hospital de niños Dr. Roberto Gilbert Elizalde en las áreas de hospitalización se evidencia que las madres adultas, jóvenes y adolescentes han dejado de amamantar a sus hijos, despertando así el interés de las autoras, de realizar un trabajo investigativo dirigido a estas madres ; para de esta manera poder evidenciar 


\section{Factores socioculturales asociados a la interrupción de la lactancia materna exclusiva en madres con niños menores de 6 meses}

Vol. 2, núm. 1., (2018)

Geny Rivera Salazar; Herlinda Espinoza Villamar; Irinay Velasco Parraga; Roció Suarez Fuentes

cuales serían los factores socioculturales que influyen en el destete temprano de la lactancia materna exclusiva antes de los 6 meses de edad, los que podrían ser: situación económica, situación familiar, estilos de vida y costumbres.

El objetivo de la presente investigación es determinar los factores socio-culturales que influyen en la interrupción de la lactancia materna exclusiva antes de los 6 meses de vida.

\section{Materiales y métodos.}

Diseño de investigación

El enfoque de la investigación es mixto, es decir cuanti-cualitativo.

- Cuantitativo: Porque nos permite cuantificar las variables de estudio

- Cualitativa: Porque nos permite estudiar los pensamientos y opiniones de las madres con respecto a la lactancia materna.

Tipo de estudio

Se trata de un trabajo de campo, descriptivo, explicativo y transversal.

- De campo: Porque nos permite obtener los datos en forma directa de las madres que son el objeto de estudio y en el lugar donde se detecta el problema planteado.

- Descriptivo: Permitió realizar un diagnóstico general con respecto a la lactancia materna. 


\section{Factores socioculturales asociados a la interrupción de la lactancia materna exclusiva en madres con niños menores de 6 meses}

Vol. 2, núm. 1., (2018)

Geny Rivera Salazar; Herlinda Espinoza Villamar; Irinay Velasco Parraga; Roció Suarez Fuentes

- Explicativo: Porque permite presentar cada una de las situaciones que se dan en las madres que brindan lactancia materna.

- Transversal: Porque nos permite seleccionar una muestra de la población, estudiar las variables simultáneamente en un determinado momento, se pudo recoger los datos de las madres durante todo un año.

\section{Modalidad de estudio}

- El Método de Análisis - Síntesis; se aplica para comprender la serie de factores que afectan a la sociedad debido a la ausencia de la lactancia materna en los niños menores de 6 meses de edad a nivel nacional análisis que permite el estudio de estos factores como lo son demográficos, situación económica, situaciones familiares, estilos de vida, costumbres. La Síntesis nos permitió descubrir las múltiples relaciones que guardan cada uno de los factores mencionados entre sí. Por lo que el análisis y la síntesis constituyen una unidad dialéctica en la actividad científica.

- El Método Inductivo - Deductivo; Nos ayudó a evidenciar de forma clara el problema sobre la falta de la lactancia en los niños menores de 6 meses de edad que afecta a la sociedad nacional, y es en base a la inducción que pasa del conocimiento de casos particulares a un conocimiento más general. Es sólo después de la investigación realizada que se deduce que la carencia de la lactancia materna en niños menores de 6 meses afecta en el desarrollo cognitivo e intelectual de los menores y es por eso que cobra gran importancia este trabajo de investigación. 


\section{Factores socioculturales asociados a la interrupción de la lactancia materna exclusiva en madres con niños menores de 6 meses}

Vol. 2, núm. 1., (2018)

Geny Rivera Salazar; Herlinda Espinoza Villamar; Irinay Velasco Parraga; Roció Suarez Fuentes

- Método de triangulación de datos: Es la combinación de los datos obtenidos en la investigación cualitativa y cuantitativa, además, de las teorías científicas que fundamentan el análisis investigativo. (11)

\section{Población y muestra}

La población se trata de madres con niños menores de 6 meses de edad, que se encuentran hospitalizados durante el año 2017.

- Para la investigación cuantitativa: La muestra fue condicionada de 130 madres que voluntariamente accedieron participar en el presente trabajo de investigación.

- Para la investigación cualitativa: Se utilizó el grupo focal para el que se escogió a 10 madres cuyos niños se encontraban ingresados en las salas de hospitalización de lactantes menores, quienes previamente firmaron un consentimiento informado, conociendo su aporte en el presente estudio.

\section{Procesamiento de datos}

Se utilizó el sistema informático de Microsoft Excel en el que se estructuró una base de datos que permitió realizar las tablas estadísticas y los gráficos.

\section{Resultados.}

1.- Contestando al primer objetivo que dice analizar la situación económica y sus repercusiones de manera que propicien el inicio o continuidad de la lactancia materna exclusiva en los primeros 6 meses de vida, los resultados que se obtuvieron fueron: 


\section{Factores socioculturales asociados a la interrupción de la lactancia materna exclusiva en madres con niños menores de 6 meses}

Vol. 2, núm. 1., (2018)

Geny Rivera Salazar; Herlinda Espinoza Villamar; Irinay Velasco Parraga; Roció Suarez Fuentes
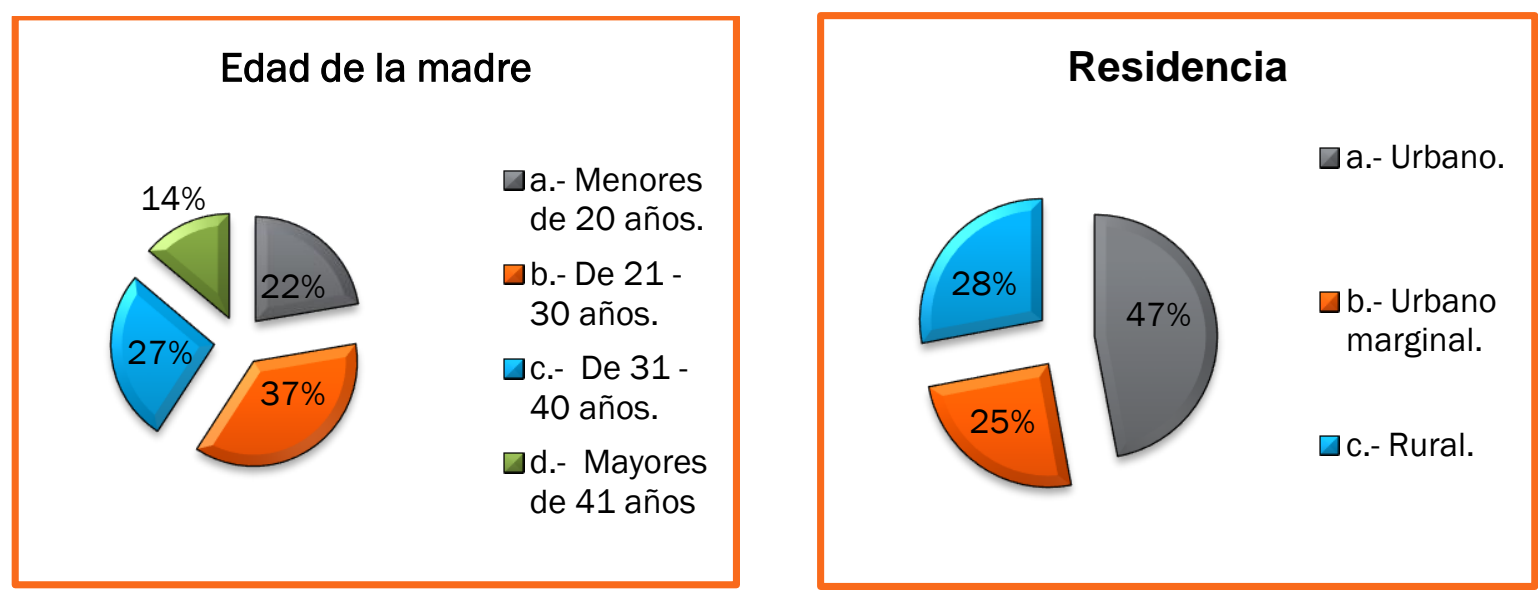

Con respecto al factor demográfico refiere en cuanto a las edades de las madres, hay una prevalencia en el abandono de la lactancia materna exclusiva entre los 21 a 40 años que corresponde al 64\%, a esto se agrega que con mayor frecuencia se ve este fenómeno en lo que respecta a la unión libre en un $49 \%$, además de acuerdo al lugar en que residen el mayor porcentaje es de $47 \%$ corresponde al sector urbano. Al analizar la subvariable situación económica, en cuanto a la actividad actual, ingresos y apoyo económico; podemos determinar que pese a recibir apoyo económico de sus parejas o familiares, el 54\% de las madres tienen la necesidad de realizar alguna actividad laboral que las obliga a salir de sus hogares, debido a que sus ingresos económicos son insuficientes y de esta manera asignan el cuidado de los menores a sus familiares. Esto demuestra que los factores económicos tuvieron sus repercusiones los mismos que influyeron en la decisión de la madre de interrumpir con la Lactancia materna exclusiva después de los 3 meses de edad del niño cuando tuvo la necesidad de reintegrarse a su área laboral. 


\section{Factores socioculturales asociados a la interrupción de la lactancia materna exclusiva en madres con niños menores de 6 meses}

Vol. 2, núm. 1., (2018)

Geny Rivera Salazar; Herlinda Espinoza Villamar; Irinay Velasco Parraga; Roció Suarez Fuentes

Estos datos, no son indiferentes a lo que expresa la OMS que: "A pesar de que se ha avanzado mucho en la conciliación de la vida laboral y la vida familiar, la mayoría de las mujeres encuentran dificultades para continuar amamantando a sus bebés cuando se reincorporan a sus trabajos". Este enunciado coincide con los resultados obtenidos que corresponden a las edades más productivas y que la mujer se incorpora a la vida laboral y además que tiene un estilo de vida del sector urbano, factores que influyen para brindar al niño lactancia materna exclusiva.

Pero a pesar de este análisis el núcleo de sentido que se obtuvo del grupo focal refleja que "La leche materna exclusiva es el alimento ideal para los niños menores de seis meses de edad por sus beneficios, nutricionales, inmunológicos y emocionales”. Las madres están conscientes de los beneficios de la lactancia materna exclusiva pero la fácil y disponibilidad de la comercialización de sucedáneos ha motivado para que forme parte de la alimentación de los niños en el momento cuando las madres se reintegran a su área laboral, y esto se lo puede explicar con las expresiones que emitieron las participantes del grupo focal como por ejemplo:

- participantes $N^{\circ} 1$ "Yo le di desde que nació 7 meses y ahora tengo un mes que le dé1 dejé de dar y le doy mixta.".

- participantes $N^{\circ} 4$ : “la niña hasta los 3 meses y medio solo leche materna y ahora que tiene 5 meses mixta".

- participantes $N^{\circ} 10$ "No yo no le he dado, pocas veces me succionó pero nada más, no le he dado".

Por lo tanto analizando el núcleo de sentido y los resultados cuantitativos, se puede fundamentar científicamente con la teoría de Madeleine Leininger se refiere a la enfermería 


\section{Factores socioculturales asociados a la interrupción de la lactancia materna}

exclusiva en madres con niños menores de 6 meses

Vol. 2, núm. 1., (2018)

Geny Rivera Salazar; Herlinda Espinoza Villamar; Irinay Velasco Parraga; Roció Suarez Fuentes transcultural como una práctica centrada en el conocimiento de los valores, creencias y prácticas de los individuos o grupos de culturas con el objetivo de proporcionar atención de enfermería en la promoción de la salud o el bienestar. "hay que promover la vida saludable que es primordial antes que los cuidados porque de ese modo hay menos gente enferma, se gastan menos recursos, se le da independencia a la gente y se mejora hacia el futuro". Por lo tanto Leinninger enfatiza que la enfermería se relaciona con la sociología porque estudia la relación entre los hombres, esta interviene en su desarrollo social su práctica se centra en el respeto a su cultura, planteando cuidados que esté acorde con su cultura. Watson es su teoría del cuidado humano manifiesta que la relación enfermera-paciente es interpersonal o intersubjetiva, ósea, una relación entre humanos en sociedad.

Como observamos en la evidencia científica demuestra que el contacto precoz con el recién nacido repercute en el buen estado de salud del pequeño y se podría decir que facilita el inicio y el mantenimiento de la lactancia materna despertando en el bebé las maniobras instintivas de búsqueda del pecho para alimentarse, lo cual disminuye el estrés de los recién nacidos, mejora el bienestar emocional de las madres y permite regular la temperatura corporal del bebé. 
Factores socioculturales asociados a la interrupción de la lactancia materna exclusiva en madres con niños menores de 6 meses

Vol. 2, núm. 1., (2018)

Geny Rivera Salazar; Herlinda Espinoza Villamar; Irinay Velasco Parraga; Roció Suarez Fuentes

2.- Identificar las diversas situaciones familiares y su impacto en el abandono de esta práctica.

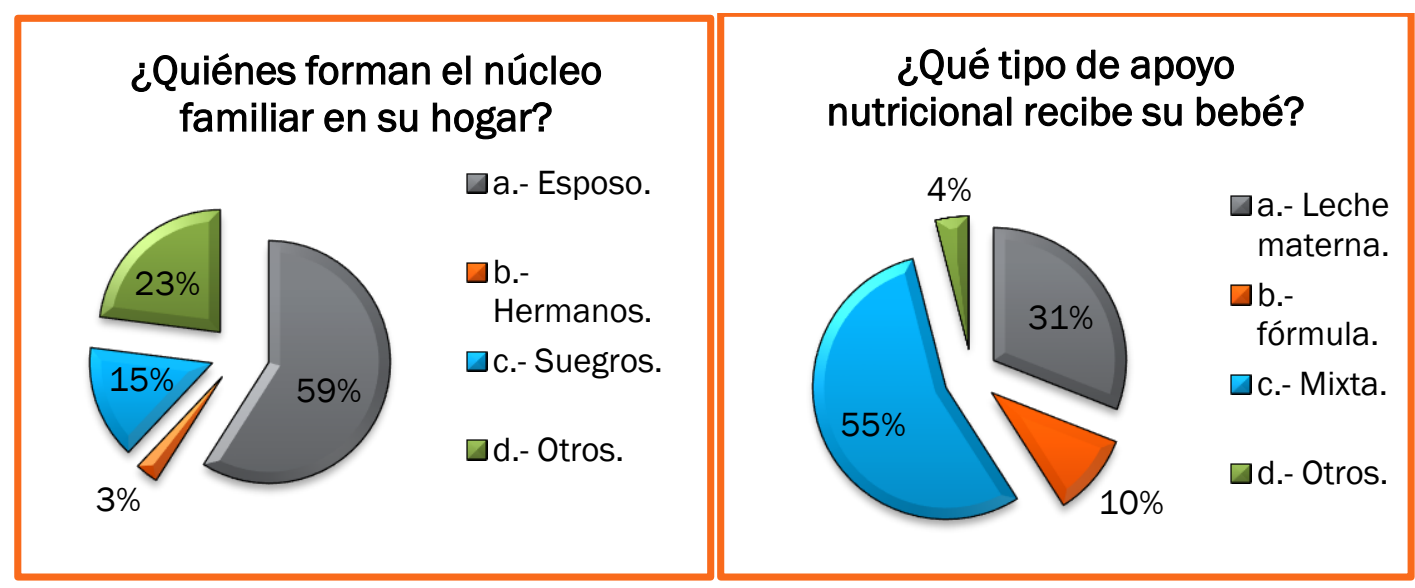

2.- Respondiendo al segundo objetivo planteado con respecto a identificar las diversas situaciones familiares y su impacto en el abandono de esta práctica, se observa que existe un 59\% de madres quienes refieren que su núcleo familiar está conformado con su esposo, lo cual podría considerarse que tienen una estabilidad familiar y, un $23 \%$ de madres indican que otras personas forman su núcleo familiar, también hay un $65 \%$ que recibieron apoyo emocional por parte de sus familiares cuando brindaban lactancia materna exclusiva, pero otros resultados preocupantes que alcanzaron al 55\% fueron de madres que tuvieron la necesidad de complementar con sucedáneos a sus niños por diferentes causas.

Según los resultados del grupo focal y al compararlos con lo que hace mención la OMS que hay varios factores sobre el abandono de la lactancia materna como madres adolescentes, pacientes hospitalizados por prematurez, cesárea e incorporación al área laboral, por lo anteriormente expuesto se menciona que existen varios factores predisponentes 1 abandona de la lactancia materna. 


\section{Factores socioculturales asociados a la interrupción de la lactancia materna exclusiva en madres con niños menores de 6 meses}

Vol. 2, núm. 1., (2018)

Geny Rivera Salazar; Herlinda Espinoza Villamar; Irinay Velasco Parraga; Roció Suarez Fuentes

Al realizar la interpretación de acuerdo al núcleo del sentido del grupo focal en lo que mencionan que: "Muchas de las veces la interrupción de la lactancia materna se debió a que su bebé se encontraba hospitalizados y/o madres que tuvieron la necesidad de reintegrarse a la vida laboral". Cabe mencionar que existen madres que tuvieron dificultades en la continuidad de esta práctica quienes tuvieron que ausentarse de su hogar en tiempos prolongados debido a factores laborales.

- Participante No. 6: "Yo le di solo leche materna, pero cuando ya entré a trabajar tuve que ayudarme con leche de formula obligatoriamente y le gustó más la leche de fórmula que la del seno y se quedó con fórmula."

- Participante No. 8: "Bueno es que cuando yo di a luz por cesaría lo primero que le dieron fue biberón y cuando yo quise dar el pecho materno no quiso coger, yo intento darle todos los días hago de todo pero ella grita, llora y se desespera poro no coge”.

- Participante No.10: "Sucedió que dejo de tomar el seno porque como estaba internada en UCIN tuvo hipoglucemia quise dar de lactar pero no quiso coger, le dieron alimentación por sonda y no pudo succionar."

Para entender estos resultados integrando el núcleo de sentido, los resultados cuantitativos y, la fundamentación científica basadas en la teórica Madeleine Leininger en la que dice: "hay que promover la vida saludable que es primordial antes que los cuidados porque de ese modo hay menos gente enferma, se gastan menos recursos, se le da independencia a la gente y se mejora hacia el futuro" así como también en su paradigma del entorno. Por lo tanto entendamos que la actividad laboral que realizan las madres de familia se constituye como un 


\section{Factores socioculturales asociados a la interrupción de la lactancia materna exclusiva en madres con niños menores de 6 meses}

Vol. 2, núm. 1., (2018)

Geny Rivera Salazar; Herlinda Espinoza Villamar; Irinay Velasco Parraga; Roció Suarez Fuentes

factor socio-económico con repercusiones en el abandono de la lactancia materna exclusiva y sobre esta perspectiva la constitución de sus familias no tuvieron gran impacto, tampoco el apoyo brindado por las mismas no fueron lo suficiente para evitar la interrupción de la lactancia materna; esto explica que la cultura de cada uno de los seres humanos influyendo en la conducta del objeto en estudio determinando su decisión de continuidad o interrupción.

\section{3.- Determinar los estilos de vida y costumbres de la madre que influyen en la interrupción de la lactancia materna.}

$\begin{gathered}\text { ¿Quién le proporcionó los } \\ \text { conocimientos previos sobre la } \\ \text { lactancia materna exclusiva para su } \\ \text { bebé? }\end{gathered}$
$\begin{gathered}\text { ab.- Familiares. } \\ \square \text { b.- Amigos. } \\ \text { enfermería. } \\ \square \text { d.- Auxiliar } \\ \text { enfermería. } \\ \square \text { a.-Ninguna. }\end{gathered}$

Respondiendo al último objetivo sobre los estilos de vida y costumbre de la madre que influyeron en la interrupción de la lactancia materna, un 45\% de las madres encuestadas indicaron que quienes les proporcionaron los conocimientos previos sobre la lactancia materna exclusiva fueron sus familiares y son ellos quienes transmiten sus creencias y costumbres alcanzando un $55 \%$.

En cuanto a los motivos que determinaron el abandono del amamantamiento fueron múltiples las respuestas en cuanto a mitos y leyenda, destacándose la incorporación a su 


\section{Factores socioculturales asociados a la interrupción de la lactancia materna exclusiva en madres con niños menores de 6 meses}

Vol. 2, núm. 1., (2018)

Geny Rivera Salazar; Herlinda Espinoza Villamar; Irinay Velasco Parraga; Roció Suarez Fuentes actividad laboral, la baja producción de leche materna se hereda, dolor a causa de pezones agrietados, pechos pequeños alcanzando un $60 \%$.

La información obtenida por el grupo focal y según como indica la OMS si se realiza una comparación entre la leche materna y los sucedáneos. La Leche Materna Exclusiva es el alimento ideal que contiene todos los nutrientes e inmunidad para los bebés, además crea una excelente relación emocional entre madre e hijo, y la alimentación alternativa como son los sucedáneos solo cubre los nutrientes básicos. El grupo focal concluye que "El conocer los beneficios de la leche materna, las madres hicieron todo el esfuerzo para brindarle su pecho a su hijo, pero por diversas situaciones patológicas tuvieron la necesidad de brindarle leche de fórmula y en la medida posible le ofrecen leche materna." Teniendo como resultado de los datos obtenido por madres en lo que hacen referencia que.

- Participante No.1: "Ahora no estoy dándole porque a mí siempre se me estaba enfermando porque ella es intolerante a la lactosa, por eso no le estoy dando mi pecho pero sé que es importante”.

- Participantes No. 8: "Yo no le doy mi niña no quiere coger desde que nació no quiso coger pecho materno porque no tengo mucha pezón hasta ahora no le doy he tratado de darle el pecho pero no quiere, por eso le doy leche de formula pero se la importancia, y en mi anterior hijo le di hasta los 8 meses y no se me ha enfermado como este niño que tengo ahora y bueno por mí me daría leche materna pero el niño no quiere coger he intentado de todo pero no quiere ahora ya no me sale mucha leche como no mama he 


\section{Factores socioculturales asociados a la interrupción de la lactancia materna exclusiva en madres con niños menores de 6 meses}

Vol. 2, núm. 1., (2018)

Geny Rivera Salazar; Herlinda Espinoza Villamar; Irinay Velasco Parraga; Roció Suarez Fuentes

tratado de sacarme con la pezonera y no me sale pero sé que la leche materna es la mejor y tiene mucho nutrientes le da inmunidad hasta que sean adultos".

Haciendo el análisis el núcleo de sentido y observando los resultados cuantitativos y, la fundamentación de la teórica Madeline "la enfermería transcultural tiene como objetivo en proporcionar cuidados significativos basados en experiencias previas de vida, dentro del contexto de su cultura, valores, creencias y estilos de vida para ayudar a mantener o recuperar la salud del ser humano y hacer frente a sus discapacidades o muerte”.

Según diversos autores enfatizan que el amplio abandono de la Leche Materna es una característica del siglo XX, según Mead Y Newton (1997), Winnikoff y Cols (1996), parece ser que influyen más los factores culturales que las capacidades biológicas de las mujeres al igual que factores socioeconómicos y familiares. Ysunsa (1992) menciona que la escolaridad y la ubicación de las familias lactantes guardan relación con el mantenimiento de la Lactancia Materna. Van Esterik y Cols (1994) enuncian como los principales factores socioeconómicos para la suspensión de la Lactancia Materna Exclusiva el ingreso creciente de las mujeres al mercado laboral, las presiones de la vida urbana de las grandes ciudades. Así mismo Edward Taylor refiere que " la cultura o civilización es todo un complejo, que incluye creencias, arte, moral, ley, costumbres y cualquier otra habilidad y hábitos adquiridos por el hombre como miembro de una sociedad". (12)

Los factores socioculturales tuvieron gran relevancia en el destete precoz antes de los seis meses de edad, no obstante la cultura y procedencia de las participantes repercutieron en la decisión de sí misma; por lo tanto en este estudio se resalta la importancia de estudiar a los seres 


\section{Factores socioculturales asociados a la interrupción de la lactancia materna exclusiva en madres con niños menores de 6 meses}

Vol. 2, núm. 1., (2018)

Geny Rivera Salazar; Herlinda Espinoza Villamar; Irinay Velasco Parraga; Roció Suarez Fuentes humanos, las relaciones entre sí y con su entorno y sobre esta perspectiva entender el comportamiento humano como un ente complejo que incluye su conducta, creencias, valores, prácticas que se aplican en las sociedades, habilidades y hábitos adquiridos por el hombre como miembro de la sociedad, así como también a la asistencia de enfermería, como lo explica Leininger que para dar cuidados es necesario planificar que estos sean culturalmente congruentes.

\section{Conclusiones.}

Considerando la importancia de la lactancia materna exclusiva como el alimento ideal para los niños menores de seis meses de edad, por sus múltiples beneficios, aportes nutricionales, inmunológicos y, emocionales, las madres de familia tuvieron la necesidad de interrumpir la lactancia materna exclusiva debido a su rol como madre de familia de contribuir con el sustento económico de su hogar tuvo que integrarse a su vida laboral, esto conllevó a la necesidad de complementar la alimentación de su hijo con sucedáneos de la leche materna.

Este estudio resalta la importancia de estudiar a los seres humanos teniendo como base la teoría de la universalidad e interculturalidad, asociada a las ciencias sociales, la misma que deberá ser congruente con su cultura proporcionando cuidados que van de la mano con sus creencias, prácticas, valores culturales individuales o grupales relacionándose entre sí y con su entorno, para entender el comportamiento de las personas como un ente complejo que incluye su conducta y hábitos adquiridos como miembro de una sociedad, así también a la enfermería como una ciencia con sustento metodológico y científico que fundamentan su práctica como lo 


\section{Factores socioculturales asociados a la interrupción de la lactancia materna exclusiva en madres con niños menores de 6 meses}

Vol. 2, núm. 1., (2018)

Geny Rivera Salazar; Herlinda Espinoza Villamar; Irinay Velasco Parraga; Roció Suarez Fuentes

explica Leininger de tener el conocimiento para planificar y brindar cuidados pero que estos sean culturalmente aceptados por el grupo humano.

\section{Bibliografía.}

1. Fescina R, De Mucio B, Díaz Rossello J, Martínez G, Serruya A. Salud Sexual y Reproductiva Montevideo: OPS; 2011.

2. Duque Jacome K, Yanez Segovia M. Factores que inciden en la duración de la lactancia materna exclusiva en dos grupos de madres de la ciudad de quito, que acuden al hospital gineceo obstétrico isidro ayora y hospital de especialidades de las FFAA durante el período de diciembre del 2015 a. Disertación previa a la obtención del titulo de Medico Cirujano. Quito: Pontificia Universidad Católica del Ecuador, Facultad de Medicina; 2016.

3. OMS. Lactancia materna exclusiva. Ginebra: Organización Mundial de la Salud; 2011.

4. OMS. Estrategia Mundial para la Alimentación del Lactante y del Niño Pequeño Singapur: Organización Mundial de la Salud; 2003.

5. World Health Organization \& UNICEF. Protección, promoción y apoyo de la lactancia natural : la función especial de los servicios de maternidad / declaración conjunta OMS/UNICEF Ginebra: OMS; 1989.

6. Belintxon-Martín M, Zaragüeta M, Adrián M, López-Dicastillo O. El comienzo de la lactancia: experiencias de madres primerizas. Anales del Sistema Sanitario de Navarra. 2011 Dic; 34(3): p. 409-418.

7. Marcela Pezzani V, Pamela Soto H, Rafael Pineda L, Elizabeth Pinilla L. Cumplimiento de la lactancia materna: Una perspectiva desde las oficinas de farmacia. Revista chilena de pediatría. 1998 Dic; 69(3): p. 99-103.

8. UNICEF. MEJORAR LA NUTRICIÓN INFANTIL El imperativo para el progreso mundial que es posible lograr New York: Fondo de las Naciones Unidas para la Infancia; 2013.

9. INEC. Encuesta Nacional de Salud y Nutrición se presenta este miércoles. Quito: Instituto Nacional de Estadisticas y Censos; 2013.

10. SEMPLADES. Plan Nacional del Buen Vivir. Quito:; 2013. 
Factores socioculturales asociados a la interrupción de la lactancia materna exclusiva en madres con niños menores de 6 meses

Vol. 2, núm. 1., (2018)

Geny Rivera Salazar; Herlinda Espinoza Villamar; Irinay Velasco Parraga; Roció Suarez Fuentes

11. Denzin N. Sociological Methods: a Source Book Chicago; 1970.

12. Piñeros B, Camacho N, Villavicencio A. Factores que inciden en la suspensión de la lactancia materna exclusiva. REVISTA ORINOQUIA. 2013; 6(1). 